\title{
Lean Practice Case for Improving Service Operations of Donuts Company ${ }^{*}$
}

\author{
Song-Kyoo Kim \\ Washington SyCip Graduate School of Business, Asian Institute of Management, Makati, Philippines. \\ Email: SKim@aim.edu
}

Received May 20 ${ }^{\text {th }}, 2013$; revised June $21^{\text {st }}, 2013$; accepted July $18^{\text {th }}, 2013$

Copyright (C) 2013 Song-Kyoo Kim. This is an open access article distributed under the Creative Commons Attribution License, which permits unrestricted use, distribution, and reproduction in any medium, provided the original work is properly cited.

\begin{abstract}
The study is based on a data set which has been corrected by the service company and analyzed using Lean Thinking tools. It provides the application of Lean Service into the actual service company. The project is intended to provide a pragmatic view of Lean application. It indicates some of the key practical factors to watch for when considering implementation aspects at each stage of the journey. Both value identification and service quality improvement were applied. The paper explores and clarifies Lean by showing how the process can be supported to add incremental value. The paper demonstrates how Lean methods can be adapted into real-world situations by applying Lean tools to the actual company. So, companies of different service industries can also learn and adapt the Lean approach more effectively.
\end{abstract}

Keywords: Lean; Lean Development; Lean Service; Lean Thinking; Service Management; Service Quality; Donuts

\section{Introduction}

Lean Thinking, or simply, Lean, describes the system now known as the Toyota Way, titled after the company responsible for its creation [1-3]. It is sometimes also known as the Toyota Production System [4] described by Taiichi Ohno [5]. The Toyota Lean principles, as presented by Womack and Jones, reveal a more holistic picture of Lean that extends beyond elimination of waste [6]. It is an iconic term for the philosophy behind the Toyota Production System of car manufacturing, also known as the thinking people system. It defines a system designed to provide the tools for people to continually improve their work and to add value to the product or service they are producing. It is based on a philosophy that abhors waste-that is, any action that does not add value to the product, or, in health care, to the patient experience [7]. A core value of the Toyota Production System is continuous improvement (Kaizen, in Japanese) applied to all processes [8]. Kaizen is often used to teach people about Lean while they improve specific processes. Later, the Lean approach to reduce wastes and add value was also

\footnotetext{
${ }^{*}$ The study is based on the research project from Innovation and Operations Laboratory (I\&O Lab.) in Asian Institute of Management. Mr. Vivek Parmer and Mr. Sneh Sagar in I\&O Lab. have been actively involved in the project.
}

brought to Western manufacturers [6].

On the other hand, Six sigma was developed at Motorola to provide a structured approach that would reduce variation in product output during the 1990s [9]. Initially, these two methodologies for dramatic improvement were seen as competitors, but in the late 1990s, a Lean Six Sigma approach was developed, combining the strengths of the two approaches [10]. Lean is often understood to be a waste reduction method where processes are examined for seven specific wastes, and the wastes are permanently removed from the process [9]. The enterprise provides what the customer wants at the moment the customer wants it. Value streams are mapped, steps are followed, and waste is eliminated. Overall, mindset within the enterprise involves a constant pursuit of perfection [6]. Although there have been a number of sources that claim to be the origins for many of the founding concepts behind Lean production, most people recognize the Toyota Production System (TPS) as the one that initially brought together all the essential ingredients required to implement a complete lean manufacturing process. The concepts included are: 1) balancing flow throughout the process, 2) pulling output to precisely match customer demand, 3) reducing inventory and waste throughout the production process, and 4) producing increased variety and complexity to what was previously possible. 
T.CO Donuts \& Coffee Company ${ }^{1}$ (hereinafter referred to as "T.CO") is a premium cafe retailer from Indonesia specializing in delivering the following goods: donuts, frozen yogurt and coffee. T.CO is well-positioned as a cafe business offering top-quality Italian coffee and chocolate beverages, complemented with a wide range of premium, innovative donuts-32 varieties in all (see Figure 1). Its donuts are light to taste, made from superior quality imported ingredients, such as Belgian chocolates, Californian almonds, Japanese matcha green tea and Italian roasted coffee. T.CO began trading in 2005 and is a fast growing donut and coffee chain in Southeast Asia with more than one hundred stores in Indonesia, eight in Malaysia, three in Singapore, and two in Shanghai, China. Contemporain Foods Inc. (under the umbrella group of companies that manufactures Oishi snack food), brought the T.CO franchise to the Philippines in March 2012. Since then, T.CO has set up shop in various shopping malls all over Metro Manila.

Lean Thinking [11] can be summarized into the two pillars that support it: 1) Continuous Improvement, and 2) Respect for People. Continuous improvement, often called Kaizen, is defined by Toyota as the basic approach to doing business [2]. More important than the actual improvements that individuals contribute, the true value of continuous improvement is in creating an atmosphere of continuous learning and an environment that not only accepts but also embraces change [11]. In addition, Lean Thinking is concerned with delivering more value for the business by increasing the velocity of output and minimizing wasteful practices by balancing process flow. Many Lean tools have been introduced, but at one time, only selected tools may apply to certain cases. In the case of T.CO., the following Lean tools will be applied to improve service quality: 1) Gemba, 2) Value Stream Mapping, 3) Process Mapping, 4) Root Cause Analysis, and 5) $5 \mathrm{~S}$ (workplace organization). These widely used techniques can be used to contribute to end-to-end process compression and improve output of services. Note that it is not the number of tools used that is crucial to success,

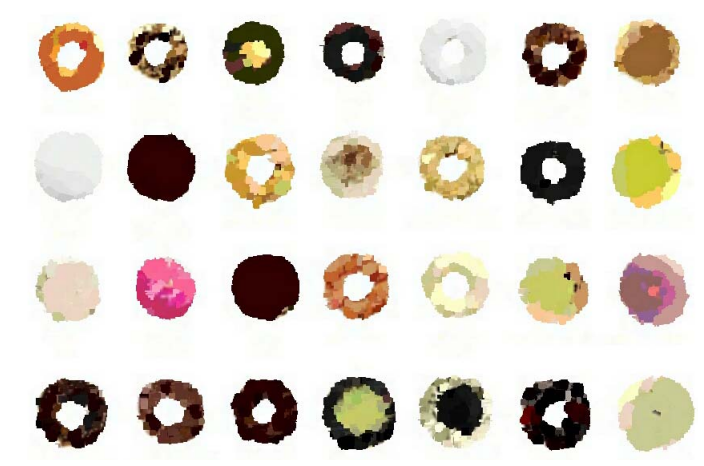

Figure 1. Various donuts flavor.

${ }^{1}$ The original research is based on the real company but the company name in the paper is made up artificially. but rather, it is the selective and appropriate deployment of tools that is important. Each technique can be deployed at varying levels of complexity and at various stages.

The scope of this paper is limited to the operations of the T.CO store located in the upscale mall on Makati City, Metro Manila. The major problem with the operations of the store is its long queues, which can be overwhelming for the customers. During peak hours, it may take up to 90 minutes for a customer to purchase two to three dozens of donuts; hence, there is an urgent need to reduce the queues attributable to the long waiting times. Turnover (or cycle time spent) by the customers who line up, pay for and step out of the store should also be addressed at the soonest possible time. On one hand, there is something highly unusual with the T.CO Donuts \& Coffee lines. Even so, this should be assessed hand-inhand with a review of the donut production processes, particularly the rotation of the available donut variants. The problem seems to be simple: the demand is high and the supply is low. However, there has been much criticism of the long queues and waiting time at T.CO, such that social media sites are teeming with negative comments.

\section{Service Operations of T.CO Donuts and Coffee}

T.CO is conveniently located on the third floor of the mall, along the same strip as various restaurants and coffee shops. Collectively, these co-tenants generate high foot traffic for the location, ensuring more customers. Owing to the open air layout of the mall, someone from the second floor can smell the sumptuous aroma of freshly baked dough from the store. First time mallgoers may begin to wonder where this amazing smell is coming from. As for the more acquainted, the presence of T.CO comes to mind and craving for T.CO donuts begins to ensue.

Unfortunately, the unusually long lines that extend from the cash counter to the back area of the store may be discouraging. Nonetheless, worth noting is the high number of people who have committed to line up and wait, and those who have chosen to wait in line will have to stay there for 30 to 80 minutes just to get their donuts. Upon entering the store, the customer will see the freshly made donuts placed in the glass display section. The customer is then greeted with the queue leading to the donut display section where someone will take their orders. If the order is readily available from the display, the staff packs the donuts in a box or prepares the same for dine-in. Once the order is prepared, it is placed in the line to the cashier for payment. The cashier then bills the customer and hands over the donuts after payment. In the event that the donuts ordered are not available on the 
display counter, the customer is informed that their order will take up to 30 minutes to prepare. If the waiting time is acceptable to the customer, the order is prepared and the customer is given a token number to identify their orders.

The store setup consists of one big station divided into three (3) sections. The donuts section, starting with the glass display fronting the customers and donut topping area right behind it welcomes customers at the entrance. The glass display counter is followed by cash counter \#1 and the yogurt display section. After the yogurt display section is the entrance door for the staff, followed immediately by cash counter \#2, and finally, the coffee section. The store floor area provides limited seating space for customers who are either waiting for their donuts or dining in to enjoy their donuts (Figure 2).

On regular days, two (2) bakers are stationed at the dressing counter: one is on standby at the frying area, and the other one works on the dressing table, going back and forth with the donut trays. Another employee is in charge of both the coffee station and the yogurt station. Finally, the remaining baking staff works at the back-end where they prepare the dough for use. Among the front line staff, two (2) employees are tasked to manage the display area, taking orders from the customers and packing or plating them accordingly. Two (2) other front liners manage each of the cash counters. The remaining frontliners rotate between taking orders and other tasks, as needed, which includes cleaning and helping with bringing the fried donuts to the dressing area.

The store hours are from 9:00 am to 1:00 am, but the staff come in as early as 6:00 am to prepare the store for the day's business. The donuts that were kept for forming the previous night are fried the next morning, such that by the time the store opens to serve the customers, these fried donuts are already available for dressing. Simultaneously, at 6:00 am, another set of bakers start the tabling work with the kneading and forming of dough. All in all, this batch will be made available for frying by 9:30 am. Dressing of donuts is at the heart of the business methodology and is largely responsible for the

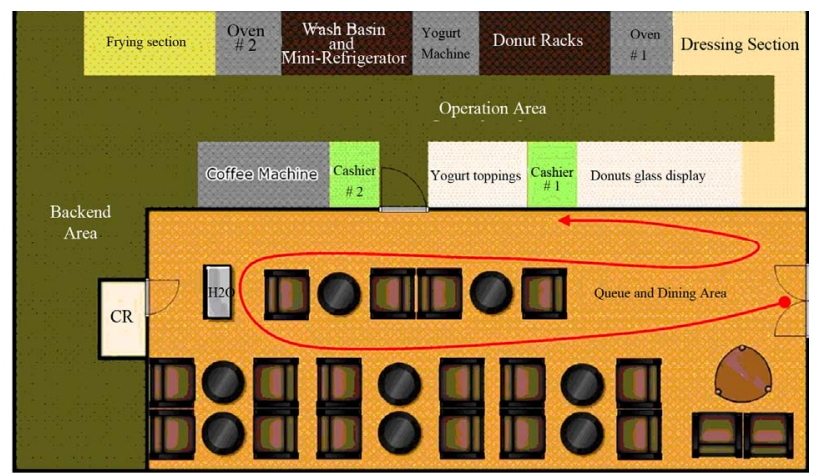

Figure 2. Present store layout (2012). popularity of T.CO donuts. Based on the sale of donuts from the display area, the master baker instructs his team of bakers to prepare which variety of donut is to be produced from thirty-two (32) different variants (Figure 1). One baker takes a tray from the oven and dresses it accordingly with each tray capacity of twelve (12) donuts. The prepared donuts generally come in two (2) different shapes, the ring and the shell. If the donut is a shell, it is first punched using a long tube and cream is injected inside through a pastry bag. After filling, one side of the donut is dipped in different sauces, such as chocolate and caramel. Next, toppings like chips and crushed almonds are drizzled on the top of each donut. Finally, the donut is transferred to the glass display trays in the display area after dressing. Sometimes, the donuts are made to rest in a temperature controlled cabinet so that the chocolate is soaked well. If the donut is ring-shaped, then the punching and cream filling is not required, resulting in reduced dressing time.

\begin{tabular}{cc}
\hline DRESSING PROCESS \\
PARAMETERS \\
\hline Dressing time per dozen \\
Dressing rate $(\mu)$ & $3[\mathrm{~min} / \mathrm{doz}]$ \\
$($ i.e., $1[\mathrm{doz}]=12[$ donuts $])$ & $20[\mathrm{doz} / \mathrm{hr}]$ \\
Dressers $(\mathrm{n})$ & 2 \\
Net output rate $(\mathrm{n} * \mu)$ & $40[\mathrm{doz} / \mathrm{hr}]$ \\
\hline
\end{tabular}

During peak hours-that is, on evenings and on the weekends-the average customer arrival rate and demand is as per the table below:

\begin{tabular}{cc}
\hline $\begin{array}{c}\text { DONUT DEMAND RATE AT } \\
\text { PEAK HOURS }\end{array}$ \\
\hline Customer arrival per hour $(\mathrm{x})$ & $38[\mathrm{order} / \mathrm{hr}]$ \\
Average order size $(\mathrm{O}),[\mathrm{doz}]$ & $1.87[\mathrm{doz} / \mathrm{order}]$ \\
Donut demand $(\lambda),[\mathrm{doz} / \mathrm{hr}]$ & $71[\mathrm{doz} / \mathrm{hr}]$ \\
\hline
\end{tabular}

The donut topping area is limited such that no more than two (2) bakers can occupy the space; otherwise, the area will be congested. When a third baker joins the dressing, the efficiency will be negatively affected (see Figure 3). In addition, all three bakers cannot simultaneously work on the dressing. As soon as one baker finishes dressing and places the tray in the oven or the display area, the other baker starts dressing another batch. The counters are designed in such a way that there is very little space between the display rack and the dressing station. During peak hours, there is a tendency for staff to bump into each other while moving from one place to another. Management admits that the space available in the area is too little; hence, the presence of 


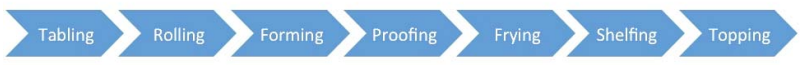

Figure 3. Donut making process.

more than one staff member in the area may obstruct the flow. Under ordinary conditions the back-end operationsstarting from tabling to frying the donuts-do not cause any delay in the production process. Only under exceptional circumstances, such as breakdown of any machinery, can there be a possible shortage of fried donuts. Bulk orders can also cause some shortage, although this is an unusual event.

The target of the T.CO project is to improve the customer turnover (i.e., long waiting lines) and enhance the system by identifying operation roadblocks and applying Lean Thinking for customer satisfaction. Lean approach provides the practical recommendations that may be directly implemented or immediately applied to the current service system. The first step in a company wide lean transformation effort is to identify the value stream. A value stream map is the tool typically used to show the flow of all materials and information, as well as the cycle times and wait times involved in making a product. A current state value stream map (vis-à-vis the ideal or desired future state) is created by visiting each process involved in making a given product in a business. Rother and Shook [12] suggest the following when creating a current state value stream map (Figure 4):

Begin with a quick walk through. Walk the entire process of material and information flow to get a sense of the flow and sequences.

Collect current-state information while walking along the actual pathways of material and information flows.

Begin at the end (shipping) and walk upstream. The downstream processes are most closely related to the customer and will set the pace for the other processes upstream.

Bring a stopwatch. You will need to collect process and flow times to calculate value-added and non-valueadded times (defined below), and record them on the current state map.

Map the entire value stream yourself; that is, if different people map different segments of the value stream, then no one will understand the whole.

Use a pencil. Start your rough sketch as you walk through the process. Plan to clean it up and transfer to a larger paper, also using a pencil. Resist the temptation to use a computer program.

\section{Lean Process: Value Identification}

The first step in a company wide lean transformation effort is to identify the value stream. Fishbone diagram is a simple and widely used tool applied not only in Kaizen

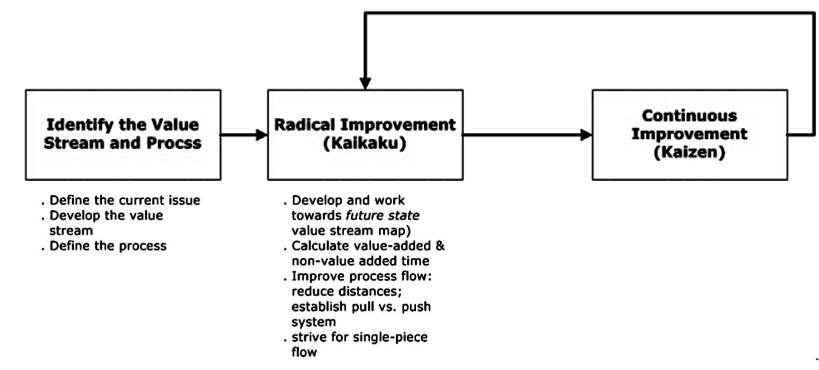

Figure 4. Value stream flowchart.

(or Kaikaku) but also in identification of the value streams. Unfortunately, Fishbone diagram presents certain limitations when it comes to identifying problems or areas for improvement, and it requires additional steps to generate new ideas. On the other hand, Root Cause Analysis (or RCA) incorporates the ideas of problem solving in its analysis. In response to a problem or defect, a team considers five (5) why's behind the problem. The reason for the number is that it is easy for people to guess wrong or weak answers unless they see the facts at the real place of the problem. In RCA, some teams create an RCA diagram of branching answers so it appears to be a more structured expansion of the Fishbone (Ishikawa) diagram. The important point of RCA is not the technique, but that it is part of the stop-and-fix root-cause problem-solving mindset ideally embedded in company culture. People are taught to become deep problem solvers-not to live with problems, but to think things through deeply. It is in many ways compatible with the "Go See" principle because issues identified may be instantly fixed after RCA activities.

\section{RCA (Expansion of Fishbone Diagram)}

After a detailed study about the system of T.CO operations, Root Cause Analysis (RCA) is applied because it is one of best tools to identify the root causes of long queues and long waiting time at the store. The root cause analysis is presented in Figure 5 below. According to the analysis, five root causes are identified: 1) monitoring of the donut display section by the T.CO headquarters in Indonesia; 2 ) wide variety of donuts (32 varieties) as one of Unique Selling Points (USP) for T.CO; 3) manual dressing of donuts as another USP of T.CO; 4) product line consisting of three items, and 5) store layout (see Figure 5). These are considered root causes, and recommendations to address them and to improve the waiting time at the queue will be discussed in the following sections.

A. Root Cause (RC) $1-$ Monitoring by the T.CO HQ: In order to monitor the quality of donuts produced and to ensure conformity with the high standards of T.CO, the donut display section of each store is monitored via CCTV camera at the T.CO headquarters in Indonesia. It 


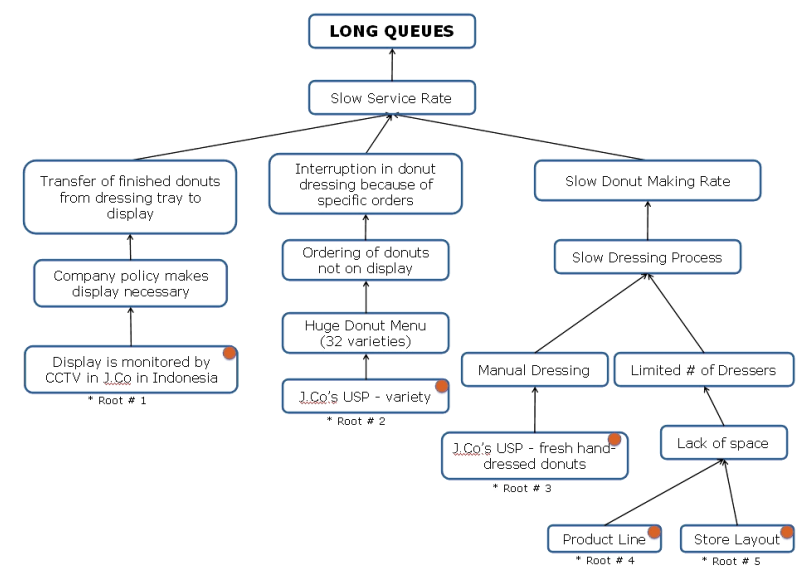

Figure 5. Root cause analysis.

is compulsory that all donuts packaged for the customers be picked from the display area only. Due to this requirement, the dressed donuts on the tray are transferred one by one onto the display tray. As the donuts are delicate and may be disfigured if mishandled, the process of transferring is very slow. If a customer orders a quantity of donuts more than those available on the display, the frostline staff picks the tray from the storage cabinet and transfers the donuts one by one onto the display tray. After he transfers the donuts to the display tray, he prepares the customer's order by picking up the donuts from the display tray one by one. This tedious process of double transfer consumes around 15 seconds and reduces the service rate of the frostline staff, thereby resulting in unnecessary queue buildup. In order to reduce inefficiency and to increase the service rate of the frostline staff, the use of a common tray for dressing as well as display is suggested. Presently, the display tray is made of glass while the dressing tray is made of plastic. Since the plastic tray is light and non-breakable, it assists in easy movement. Hence, replacing the plastic dressing tray with glass tray is not an option. We recommend, instead, replacing both the display tray and the dressing tray with fiberglass trays which are lightweight, transparent and visibly pleasing. This would ease the operation process and remove the double time consumed in packaging the donut. The time saved will be approximately 15 seconds per dozen donuts sold.

B. Root Cause 2-Large selection of Donuts on Menu (32 varieties): The T.CO donut menu consists of 32 varieties of donuts. However, the display area can only accommodate 16 trays; hence, at any given time, only a maximum of 16 varieties of donuts are displayed. The master chef randomly decides what donut varieties to make, and it is only when a customer orders a donut not on display that the dresser prepares the requested donut variety, after finishing the batch which he is presently working on. This causes delay in the dressing of the regular donut, and at times, results in shortage of the regular donut on display. 32 donuts offered by the shop is part of its standard offering, as per company policy. However, the menu is divided in half and rotated alternately by making 16 varieties of donuts available every other day, as recommended. This reduces the operational hassle of preparing all 32 varieties every day, and it reduces the disruptions in the regular donut dressing process. In case a customer wants to order a specific variety and the variety is not available on that day, he/she may place the order in advance or wait in the store for a specified period (e.g., one hour) to collect his/her order. This will give the dressers sufficient time to prepare the requested donut variant without interrupting the regular donut-making process.

C. Root Cause 3-Manual dressing of donuts: Most high-volume donut stores have an automated donut dressing system, but freshly-made donuts, artistically handcrafted in front of the customers, is another USP of T.CO. This drives the volume of the donuts but at the same time slows down the donut-dressing rate. The output rate also varies depending upon the skills of the dresser. However, since this is the unique selling point of T.CO donuts, the manual dressing of donuts is required as per HQ policy. The dresser needs to get a tray of fried donuts from the temperature-controlled cabinet for dressing, which takes around 15 seconds. The actual dressing of the donuts takes 2.5 minutes. The dresser then goes back to put the dressed donuts in the temperature-controlled cabinet, taking an additional 15 seconds.

D. Root Cause 4-Product line consisting of three items: The standard product lineups of T.CO are donuts, yogurt and coffee, and donuts generate the maximum sales, followed by coffee. Yogurt, however, is hardly sold in any of the three stores in Philippines. The yogurt line covers nearly 20 percent of the front-end display area of the store because it is part of the company policy. Even though removing their yogurt lines may be the candidate recommendation to increase the donut dressing rate, this would be difficult to apply because the final decision lies with T.CO top management in Indonesia.

\begin{tabular}{ccc}
\hline $\begin{array}{c}\text { COMPARISON OF } \\
\text { PROCESS }\end{array}$ & Current & Recommended \\
\hline $\begin{array}{c}\text { Dressing time per } \\
\text { dozen }\end{array}$ & $3[\mathrm{~min} / \mathrm{doz}]$ & $3[\mathrm{~min} / \mathrm{doz}]$ \\
Donut dressing rate $(\mu)$ & $20[\mathrm{doz} / \mathrm{hr}]$ & $20[\mathrm{doz} / \mathrm{hr}]$ \\
$\begin{array}{c}\text { Dressers }(\mathrm{n}) \\
\begin{array}{c}\text { Net donut output rate } \\
(\mathrm{n} \cdot \mu)\end{array}\end{array}$ & 2 & 3 \\
\hline
\end{tabular}

However, if management decides not to remove the yogurt product line due to the company policy, then 
removing the counter displaying available toppings may be recommended. Once this space is cleared, this area can be utilized for an additional dresser, thereby improving the donut-dressing rate.

E. Root Cause 5-The store layout: The present layout (see Figure 2) illustrates a congested working area. The space available between the front display row and the back utility row is small and leads to congestion, when more than one person is mobile. Because of limited space, free movement is restricted, which leads to wastage of time, i.e., the time that dressers take to transfer the finished donut trays to the temperature-controlled cabinet. To minimize such time wastage, a slight modification in the store layout is recommended.

The present layout has one wide sitting row along the wall, one small sitting row in the center of the store and the display and billing counter. Because of long queues, the middle row becomes surrounded by persons standing in the queue, making it uncomfortable for the customers sitting there. Hence, removal of the double-sitter middle row is recommended, as well as moving the display counters outwards. By moving the display counters outwards, sufficient space is provided between the display row and the utility row for free movement of the employees and materials. Thus, the time wasted in moving from the dressing table to the temperature-controlled cabinet can be reduced, improving the donut-dressing rate. The enhanced store layout would also allow T.CO to utilize three dressers during peak load hours. Notably, this alteration would require the store to be shut down for 2 to 3 days for renovation and would also require monetary expense. The resulting increase in the donut dressing rate, however, would lead to reduced queue length and a significant improvement in customer satisfaction.

\section{Lean Process: Service Quality Improvement}

Kaikaku means that an entire business is changed radically, and it is most often initiated by management since the change as such and the result will significantly impact business. Kaikaku can be initiated by external factors, such as new technology or market conditions. Kaikaku may also be initiated when management see that ongoing Kaizen work is beginning to stagnate and no longer provides adequate results in relation to the effort. Recommendations for T.CO are meant to impact customer satisfaction by directly troubleshooting the current service delivery, which is part of Kaikaku.

Kaizen (Improvement) is the idea of continuous improvement of the process, of every step in the process through relentless evaluation. The improvement could come in the form of resources, time, or quality. This should not be confused with a Kaizen event, where a single workstation or process is the focal point and pro- ject improvement is not part of the daily routine. Kaizen is meant to be realized every day. It should be part of everyone's daily routine to find ways to improve the process. Some recommendations are the guidelines for maintaining the improvement continuously, and that is the part of Kaizen application.

Gemba is to find the facts needed in order to make correct decisions, build consensus, and achieve goals at one's best speed [13]. "Go See" is a principle not found in many management cultures. This principle is described as critical and fundamental. In the internal Toyota Way 2001, it is highlighted as the first factor for success in continuous improvement. "Go See" shows up repeatedly in Toyota manager quotes and in Toyota culture and habits [14]. The operational issues of the T.CO project are identified by observing the store in Makati, and it has been developed based on the observable facts.

\subsection{Reverse Process Mapping}

Process Map illustrates the workflow, which normally encapsulates the more complete operation. These process inputs are further defined, keeping in mind the goal of transforming inputs into predefined outputs. This enables product and service designers to envision how the intricacies of the critical processes affect the finished project. Also known as process charts, it has become much more prevalent and understood in the business world in recent years. Once created by a bricklayer to increase efficiency, process mapping is now being used by companies worldwide and individuals alike. Process maps can be used in every aspect of life or business.

In the T.CO project, the different type of process mapping is applied (see Figure 6). As opposed to breaking down the detailed processes from the main process, reverse process mapping which consider one chuck of process that not effects of the main process. The approach gives a clearer view of the main process. Note that not all processes are lumped together, but rather, the processes mapped are only those that are not bottlenecks nor parts of the critical activities

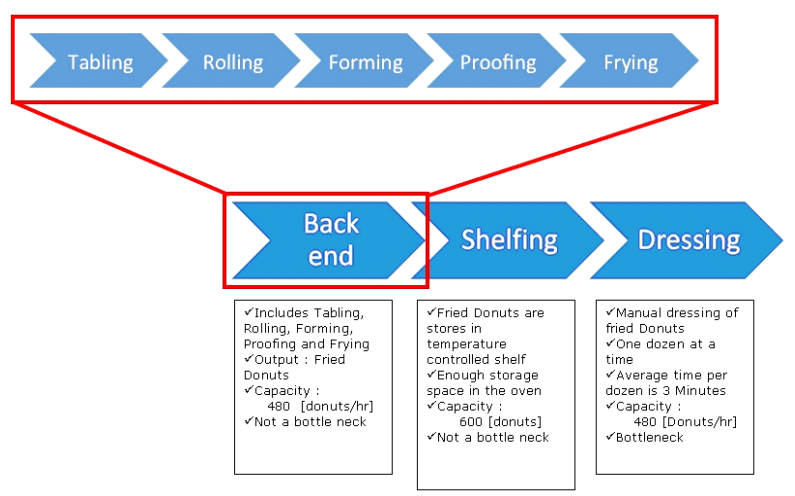

Figure 6. Reverse process mapping. 
The flexibility of process mapping in Lean Thinking gives mangers a clear view of workflow and helps them see issues in the system easily. It also transforms the process into a "single-piece flow", which is a major objective in lean service process, apart from coming up with more innovative recommendations.

\subsection{Selected Recommendations}

Recommendation \#1 (from RC \#3): Under the present situation, the output of both the dressers taken together is 480 donuts per hour. The dressing table remains idle for 30 seconds per cycle. During peak hours, the output rate of dressed donuts would increase considerably if the dressing table is fully utilized (i.e., no idle time). Furthermore, if the dresser is assigned with the single task of dressing, and one of the assistant staff is asked to bring the fried donuts and place the dressed donuts in the temperature - controlled storage unit (instead of the dresser), the donut dressing rate would increase to 576 donuts per hour - an increase of twenty percent $(20 \%)$ over the previous output. This increase would reduce the average waiting time considerably.

\begin{tabular}{ccc}
\hline $\begin{array}{c}\text { COMPARISON OF } \\
\text { PROCESS }\end{array}$ & Current & Recommended \\
\hline Dressing time per dozen & $3[\mathrm{~min} / \mathrm{doz}]$ & $2.5[\mathrm{~min} / \mathrm{doz}]$ \\
Donut dressing rate $(\mu)$ & $20[\mathrm{doz} / \mathrm{hr}]$ & $20[\mathrm{doz} / \mathrm{hr}]$ \\
Dressers $(\mathrm{n})$ & 2 & 2 \\
Net donut output rate $(\mathrm{n} \cdot \mu)$ & $40[\mathrm{doz} / \mathrm{hr}]$ & $48[\mathrm{doz} / \mathrm{hr}]$ \\
\hline
\end{tabular}

Recommendation \#2 (from RC \#5): In the revised layout (see Figure 7), the new dressing table should be designed in such a way that the toppings container is placed on top of the table, together with the liquid dips for dressing. This would further reduce the dressing time by 10 to 15 seconds per cycle.

\subsection{Overall Recommendation Summary}

From RCA (see Figure 5), recommendations are pro-

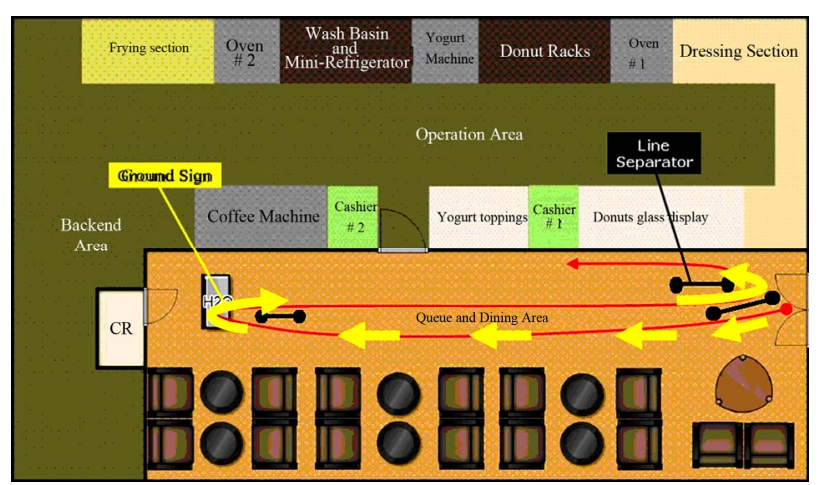

Figure 7. Proposed store layout. posed to improve or solve the root causes even though some recommendations may face certain limitations when it comes to actual implementation. The recommendations aimed at solving the root causes are shown at Table 1.

\section{Conclusions}

T.CO project is intended to provide a pragmatic view of Lean application. It indicates some of the key practical factors to watch for when considering implementation aspects at each stage of the journey. Both value identification and service quality improvement were defined, and useful enabling tools such as RCA and Process Mapping have been intelligently and appropriately deployed with Lean Thinking. The paper explores and clarifies Lean by showing how the process can be supported to add incremental value. Success depends on the essential

Table 1. Overall recommendation.

\begin{tabular}{|c|c|c|}
\hline $\begin{array}{l}\text { Root Cause } \\
\text { (RC) }\end{array}$ & Recommendation & Expectations \\
\hline $\begin{array}{l}\text { RC \#1: All } \\
\text { donuts to be } \\
\text { put on display } \\
\text { before packing } \\
\text { for customers }\end{array}$ & $\begin{array}{l}\text { Replace the donut dressing } \\
\text { tray and the display tray with } \\
\text { the same kind of transparent } \\
\text { fiber tray that can serve the } \\
\text { purpose of dressing as well as } \\
\text { display. }\end{array}$ & $\begin{array}{l}\text { Significant improvement } \\
\text { in service rate, as the } \\
\text { time required for } \\
\text { transferring the donuts } \\
\text { on the display trays and } \\
\text { for cleaning the display } \\
\text { trays are reduced. }\end{array}$ \\
\hline $\begin{array}{l}\text { RC \#2: Large } \\
\text { selection of } \\
\text { donuts on } \\
\text { menu } \\
\text { (32 donuts) }\end{array}$ & $\begin{array}{l}\text { Rotate } 16 \text { varieties on } \\
\text { alternate days. All donuts on } \\
\text { the menu can be displayed at } \\
\text { once. If a customer wants to } \\
\text { order a variety that is not } \\
\text { present on the today menu, he } \\
\text { can order in advance } \\
\text { (via phone or internet) before } \\
\text { visiting the store. }\end{array}$ & $\begin{array}{l}\text { Prioritization process of } \\
\text { the donuts to be } \\
\text { prepared will be } \\
\text { streamlined, reducing } \\
\text { the disruption of normal } \\
\text { donut dressing. }\end{array}$ \\
\hline
\end{tabular}

Dresser should only be dressing the donuts and

RC \#3: $\quad$ should not be involved in

Manual Dressing Process transferring the tray from the dressing table to the storage unit. This task may be done by any other front end staff.

RC \#4: Slow moving product line (Yogurt)

Remove the display counter of yogurt and utilize the

This will result in reduction of 30 seconds per dozen in the donut-making time. With the revised rate there will be an increase of $20 \%$ in the donut dressing rate and increased service rate, resulting in a shorter queue. space generated to minimize increase the donut obstructive movement in the dressing rate from 480 to donut-making area, facilitating 720 donuts per hour, hassle free movement. Add thereby reducing the one additional dresser. queue length.

RC \#5: Store layout cpntributing to congested working area
Shift the front line counters (display section) outward, thus increasing space for free movement.
Free movement in the donut-making area, increased efficiency and reduced time wastage caused by the obstruction from minimal space. 
balance of process, people and technology, which is necessary to sustainable deliver the highest levels of process performance. As Lean continues to be applied, there may be more success stories but it may not deliver real business benefits because of various circumstances. The real benefits may be limited for T.CO story but the research is still a clear example that Lean is a management philosophy aligns well with inclusive and effective management principles.

\section{REFERENCES}

[1] T. Fujimoto, "The Evolution of a Manufacturing System at Toyota," Productivity Press, New York, 1999.

[2] Toyota Co., "Toyota Way," Toyota Motor Company, 2001.

[3] J. Womack and D. T. Roos, "The Machine That Changed the World," Harper Perennial, 1990.

[4] W. J. Hopp and M. L. Spearman, "Factory Physics," 2nd Edition, Irwin McGraw-Hill, Boston, 2001.

[5] T. Ohno, "Toyota Production System: Beyond LargeScale Production," Productivity Press, New York, 1988.

[6] J. P. Womack and D. T. Jones, "Beyond Toyota: How to
Root Out Waste and Pursue Perfection," Harvard Business Review, Vol. 74, No. 5, 1996, pp. 140-158.

[7] C. Furman and R. Caplan, "Applying the Toyota Production System: Using a Patient Safetyalert System to Reduce Error," Joint Commission Journal on Quality and Patient Safety, Vol. 33, No. 7, 2007, pp. 376-386.

[8] J. Liker, "The Toyota Way," McGraw-Hill, New York, 2004.

[9] F. W. Breyfogle, "Implementing Six Sigma," John Wiley and Sons, Inc., New York, 1999.

[10] M. George, "Lean Six Sigma: Combining Six Sigma Quality With Lean Speed," McGraw-Hill, New York, 2002.

[11] J. Womack and D. T. Jones, "Lean Thinking," Free Press, New York, 1996.

[12] M. Rother and J. Shook, "Learning to See: Value Stream Mapping to Create Value and Eliminate Muda," The Lean Enterprise Institute, Brookline, 1999.

[13] P. Varkey, et al., "Basics of Quality Improvement in Health Care," Mayo Clinic Proceedings, Vol. 62, 2007, pp. 735-739.

[14] J. Liker and M. Hoseus, "Toyota Culture: The Heart and Soul of the Toyota Way," McGraw-Hill, New York, 2008. 